\title{
Estudio Clínico Comparativo entre Colutorio de p-clorofenol y peróxido de hidrógeno con Colutorio de Clorhexidina al $0.12 \%$ en el Crecimiento de Placa Microbiana y Gingivitis
}

\author{
Mouthwash Comparative Study between p-chlorophenol and hydrogen peroxide \\ with Chlorhexidine Mouthwash $0.12 \%$ in the Growth of \\ Microbial Plaque and Gingivitis
}

Jaña PD ${ }^{1}$, Yévenes $L^{2}$, Rivera AS $^{3}$

\begin{abstract}
RESUMEN
Se formuló un colutorio a base de p-clorofenol alcanforado y peróxido de hidrógeno, y considerando que no se disponían de antecedentes de eficacia y seguridad del colutorio, se realizó un ensayo clínico para medirlas utilizando un modelo de formación de placa microbiana y gingivitis en un período de cuatro días, como control positivo se uso un colutorio de clorhexidina al $0.12 \%$. Participaron 26 sujetos en un estudio doble ciego cruzado y con distribución aleatoria. El colutorio en base a p-clorofenol alcanforado y peróxido de hidrógeno y el de clorhexidina tuvieron un similar efecto en el control de la formación de placa y antigingivitis. No hubo diferencias entre los acontecimientos adversos observados para el colutorio y el control. Por consiguiente, el colutorio en estudio podría ser una alternativa para el control químico de la placa microbiana y gingivitis en el tratamiento de la enfermedad periodontal.
\end{abstract}

Rev. Clin. Periodoncia Implantol. Rehabil. Oral Vol. 3(2); 65-68, 2010.

Palabras clave: Colutorio, p-clorofenol alcanforado, peróxido de hidrógeno, clorhexidina, placa microbiana, gingivitis.

\begin{abstract}
A camphorated p-chlorophenol and hydrogen peroxide-based mouthwash was prepared, and a clinical trial to measure the efficiency and safety records of the mouthwash -considering the lack of them- was carried out using a formation model of microbial plaque and gingivitis over a 4-day period, with a $0.12 \%$ chlorhexidine mouthwash as positive control. Twenty-six individuals participated in the randomized double-blind crossover study. The camphorated p-chlorophenol and hydrogen peroxide-based and chlorhexidine mouthwash had a similar effect in controlling the formation of plaque and antigingivitis. There was no difference between the adverse events observed for the mouthwash and the control. Therefore, the mouthwash in study might be an alternative to the chemical control of microbial plaque and gingivitis in the periodontal desease treatment.
\end{abstract}

Rev. Clin. Periodoncia Implantol. Rehabil. Oral Vol. 3(2); 65-68, 2010.

Key words: Mouthwash, camphorated p-chlorophenol, hydrogen peroxide, chlorhexidine, microbial plaque, gingivitis.

\section{INTRODUCCIÓN}

El control de la placa microbiana y de sus efectos sobre la salud bucal es una preocupación constante entre los especialistas e investigadores sobre el tema. Lo más comúnmente usado es el control mecánico mediante cepillado y elementos coadyuvantes; sin embargo tiene resultados muy limitados pues depende en gran medida de la destreza manual del paciente. Por otro lado, algunos compuestos químicos han demostrado ser efectivos en el control de la placa microbiana mejorando los efectos del cepillado dental. El "gold standard" en cuanto a colutorios antiplaca microbiana y gingivitis es la clorhexidina $^{(1)}$. Existen una amplia gama de agentes antibacterianos en odontología que no han sido formulados como colutorio, pero que cada uno de ellos, o combinados, podrían ser eficaces, como los derivados fenólicos y el peróxido de hidrógeno.

El fenol o ácido fénico es un antiséptico clásico para controlar microorganismos y constituye la base de varios compuestos usados en odontología. Actúa en el citoplasma bacteriano y por ello es muy eficaz aún en concentraciones bajas del $1 \%$ ó $2 \%$. Suele diluirse en alcanfor y utilizarse en endodoncia como solución de fenol alcanforado $30 \%$ fenol, $60 \%$ alcanfor y $10 \%$ alcohol etílico) ${ }^{(2)}$. Con la alcanforación se busca la obtención de un producto menos cáustico, por la lenta liberación del fenol. El fenol alcanforado es el menos tóxico de los compuestos fenólicos y posee un excelente efecto antimicrobiano. El monoclorofenol es un derivado del fenol que tiene tres isómeros de los cuales el más eficaz es el p-clorofenol ${ }^{(3)}$. Es conocido el poder antibacteriano y antiséptico del p-clorofenol alcanforado y ha sido usado desde hace muchos años en endodoncia ${ }^{(4,5)}$.

Los derivados fenólicos en su mecanismo de acción desarrollan una serie de eventos moleculares que producen la muerte bacteriana. En altas concentraciones actúan como bactericida destruyendo la pared celular y precipitando proteínas. En bajas concentraciones, y con mayor peso molecular, provocan la muerte bacteriana por inactivación de sistemas enzimáticos y filtración de metabolitos esenciales a través de la pared celular ${ }^{(6)}$.

El peróxido de hidrógeno es usado al $3 \%$ debido a sus propiedades como antiséptico general, como desinfectante y a su acción efervescente. En odontología es utilizado como compuesto blanqueador a diferentes concentraciones en el tratamiento de la pericoronaritis y en procesos gingivales agudos. Su mecanismo de acción esta centrado en la reacción de iones superoxidantes y radicales libres hidroxilos que atacan la membrana lipídica, ADN y otros componentes celulares de las bacterias. Dependiendo de la concentración puede destruir a la mayoría de las bacterias, incluyendo esporas ${ }^{(7,8,9)}$.

En la búsqueda de sustancias químicas para el control de la placa microbiana, en el Área de Química de la Facultad de Odontología de la Universidad de Chile se desarrolló un colutorio compuesto de p-clorofenol alcanforado y peróxido de hidrógeno que permitiría el

1. Cirujano Dentista. Universidad de Chile. Chile.

2. Profesor Adjunto. Área de Química, Facultad de Odontología. Universidad de Chile. Chile.

3. Profesora Asociada. Área de Periodoncia, Facultad de Odontología. Universidad de Chile. Chile.

Correspondencia autor: Sonia Rivera Álvarez. soniarivera27@hotmail.com. Av. Santa María 0596, Providencia, Santiago. Chile.

Financiamiento: Facultad de Odontología, Universidad de Chile. Chile. Trabajo recibido el 15/11/2009. Aprobado para su publicación el 06/09/2010. 
control de la placa microbiana y de la gingivitis. Dado que no hay estudios de citotoxicidad de estos antisépticos en enjuagatorios, se desarrolló un estudio basado en la citología exfoliativa para analizar el efecto del colutorio sobre células de la mucosa oral(10). La morfología nuclear se clasificó en 5 tipos: Normal, Picnosis, Cariorrexis, Cariolisis y Queratinización ${ }^{(11)}$, y después de la aplicación del enjuagatorio y control no se observaron diferencias significativas en el conteo celular. Los resultados indican que el colutorio a base de p-clorofenol alcanforado y peróxido de hidrógeno no generaría un efecto tóxico evidenciable con las metodologías utilizadas en este trabajo. Se ha visto que los componentes a otras concentraciones utilizadas en odontología ( $p$-clorofenol alcanforado $33 \%$ y peróxido de hidrógeno $3-35 \%$ ) sí provocan citotoxicidad ${ }^{(12,13,14)}$. Sin embargo, las cantidades usadas en esta formulación son muy inferiores a las otras presentaciones. Además, se considera que el peróxido de hidrógeno es seguro a concentraciones de $0.1 \%{ }^{(15)}$. Dadas las diluciones utilizadas en el enjuagatorio, es esperable no encontrar alteraciones en las células de la mucosa oral por su uso.

El presente estudio tiene como objetivo comparar los efectos de este colutorio a base de $\mathrm{p}$-clorofenol alcanforado al $0.03 \%$ y peróxido de hidrógeno al $0.04 \%$ con un colutorio de clorhexidina al $0.12 \%$ en el control de la placa microbiana y gingivitis.

\section{MATERIAL Y MÉTODO}

En un estudio, doble ciego, cruzado y con distribución aleatoria de tratamientos, se reclutaron 26 individuos que, distribuidos aleatoriamente y en idéntica proporción, fueron tratados con los colutorios experimental y control ( $p$-clorofenol alcanforado - peróxido de hidrógeno y clorhexidina). Para asegurar el control doble ciego los colutorios (control y experimental) fueron envasados en frascos ámbar de $50 \mathrm{ml}$ y coloreados del mismo color.

Los sujetos eran todos sanos, mayores de 18 años y accedieron a participar en el estudio firmando el correspondiente "Consentimiento Informado". Para ser incluidos en el estudio debían tener como mínimo 20 dientes, no presentar signos de enfermedad periodontal ni haber recibido tratamiento antimicrobiano en los últimos 6 meses; tampoco estar embarazada, padecer enfermedades crónicas o estar en tratamiento con fármacos que inducen agrandamientos gingivales.

Tras una evaluación clínica basal en el Edificio Clínico de la Facultad de Odontología de la Universidad de Chile, los individuos recibieron una completa profilaxis y se les controló la higiene bucal durante dos semanas (tres cepillados diarios con técnica de Bass y un cepillo de consistencia suave).

A continuación se realizó la primera fase experimental en la que durante cuatro días se suprimió toda medida de higiene bucal y los individuos fueron tratados con uno de los colutorios previamente asignado de acuerdo con una tabla de aleatorización.

\section{Distribución Aleatoria de los Tratamientos}

A los sujetos en estudio se les adjudicó un número consecutivo y se les entregó el tratamiento que correspondía a dicho número para cada una de las dos fases experimentales. Los sujetos fueron distribuidos aleatoriamente y en idéntica proporción a las secuencias de tratamiento.

Al quinto día se evaluaron los índices Gingival y de Placa Microbiana, como también los acontecimientos adversos.

Finalizada esta primera fase todos los sujetos entraron en una fase de blanqueo de dos semanas que incluyó una completa profilaxis y un refuerzo de la higiene.

Luego se realizó la segunda fase experimental en la cual los sujetos interrumpieron nuevamente por un periodo de cuatro días la higiene bucal y fueron tratados con el segundo colutorio, distinto al empleado en la primera fase. En el quinto día se midieron el índice Gingival, de Placa y se consignaron los acontecimientos adversos.

Durante cada fase experimental los sujetos realizaron enjuagatorios bucales con $10 \mathrm{ml}$ durante un minuto dos veces al día, después del desayuno y antes de dormir, y no se les permitió ingerir alimentos o líquidos en los 30 minutos posteriores al uso del producto.

Para la determinación del estado gingival se aplicó el índice de Loe y Silness ${ }^{(16)}$ y para evaluar la placa microbiana se uso el índice de Quigley-Hein modificado por Turesky ${ }^{(17)}$, ambos índices fueron valorados por un único operador calibrado.

Acontecimiento adverso. Se define como "Cualquier suceso médico adverso que pueda presentar un paciente o sujeto de investigación clínica al que se ha administrado un producto farmacéutico y que no tiene necesariamente una relación causal con dicho tratamiento". El registro de eventos adversos se realizó mediante anamnesis, examen clínico y consignación en la "Ficha de Recepción" de acontecimientos adversos.

En relación con el análisis estadístico se aplicaron el t-test de Student para muestras pareadas y el test de Wilcoxon. Se fijó un intervalo de confianza de $95 \%$ aceptando diferencias estadísticamente significativas cuando $p<0.05$. Para el análisis de los acontecimientos adversos se usó el test Chi-cuadrado.

\section{RESULTADOS}

De los 26 sujetos en estudio 9 eran mujeres, 17 varones y todos completaron la experiencia usando correctamente los dos tratamientos.

Índice de Placa. La cantidad de placa microbiana después del uso de los colutorios se determinó a través del índice de Quigley \& Hein modificado por Turesky, obteniéndose para p-clorofenol alcanforadoperóxido de hidrógeno un promedio en el índice de Placa de 2.34 y 2.20 para clorhexidina, no apreciándose diferencias estadísticamente significativas entre ambos fármacos $(p=0.128)$.

En la Figura 1 se puede observar los valores promedios del índice de Placa de cada uno de los individuos después del tratamiento por cuatro días con cada uno de los colutorios.

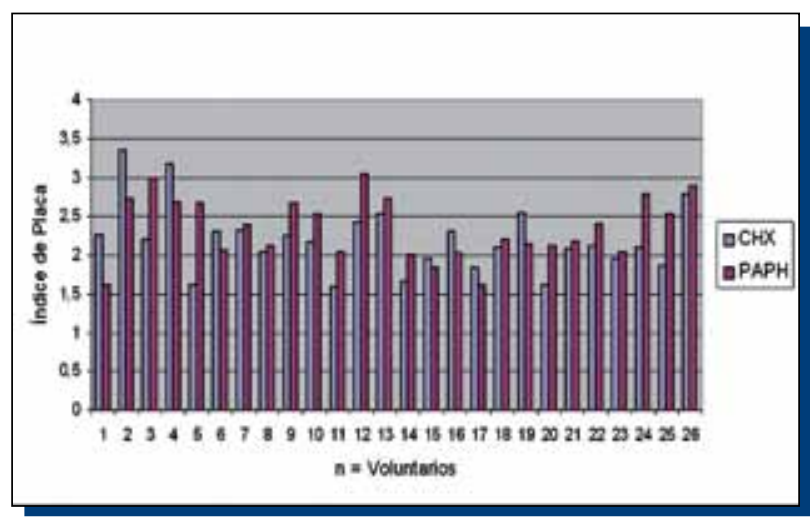

Figura 1. Índice de Placa promedio de cada voluntario después de los cuatro días de tratamiento con cada colutorio. CHX: clorhexidina. PAPH: p-clorofenol alcanforadoperóxido de hidrógeno.

Al comparar la efectividad de ambos colutorios en el control de placa en individuos del mismo sexo no se encontró diferencias estadísticamente significativas (mujeres $p=0.216$, hombres $p=0.081$ ). Por arcada, el índice fue significativamente superior en el maxilar superior $(p=0.046)$ para la clorhexidina. En las superficies dentarias, el colutorio p-clorofenol alcanforado-peróxido de hidrógeno fue más eficaz en superficies palatinas o linguales $(p=0.025)$. Los efectos de la clorhexidina fueron mejores en superficies vestibulares $(p=0.001)$ y en el sector posterior $(p=0.025)$. En la Tabla 1 se puede observar un resumen de estos resultados.

Tabla 1. Índice de Placa, tabla resumen

\begin{tabular}{|l|c|c|c|c|c|c|c|c|c|}
\hline \hline \multirow{2}{*}{} & \multicolumn{2}{|c|}{ Sexo } & \multicolumn{2}{c|}{ Arcada } & \multicolumn{2}{|c|}{ Superficie } & \multicolumn{2}{|c|}{ Sector } \\
\cline { 2 - 10 } & TOTAL & $\mathbf{M}$ & $\mathbf{H}$ & $\mathbf{S}$ & $\mathbf{I}$ & $\mathbf{V}$ & $\mathbf{P} / \mathbf{L}$ & $\mathbf{A}$ & $\mathbf{P}$ \\
\hline $\mathbf{C H X}$ & 2.200 & 2.147 & 2.229 & 2.125 & 2.269 & 2.340 & 2.061 & 2.167 & 2.234 \\
\hline PAPH & 2.348 & 2.267 & 2.391 & 2.304 & 2.393 & 2.811 & 1.885 & 2.260 & 2.442 \\
\hline $\mathbf{p}$ (t-test) & 0.128 & 0.216 & 0.081 & 0.046 & 0.108 & 0.001 & 0.025 & 0.160 & 0.025 \\
\hline
\end{tabular}

CHX: colutorio de clorhexidina, PAPH: colutorio a base de p-clorofenol alcanforado y peróxido de hidrógeno, M: mujeres, H: hombres, S: arcada superior, I: arcada inferior, V: superficie vestibular, P/L: superficie palatino/lingual, A: sector anterior, P: sector posterior.

\section{Índice Gingival}

Los resultados del índice Gingival determinados aplicando los criterios de Loe \& Silness indican que no se encontraron diferencias estadísticamente significativas entre ambos colutorios $(p=0.378)$. Los promedios para $\mathrm{p}$-clorofenol alcanforado-peróxido de hidrógeno fueron 0.107 y 0.099 para clorhexidina. 
En la Figura 2 se puede observar los valores promedio del índice Gingival de cada sujeto después de cuatro días de tratamiento con cada colutorio.

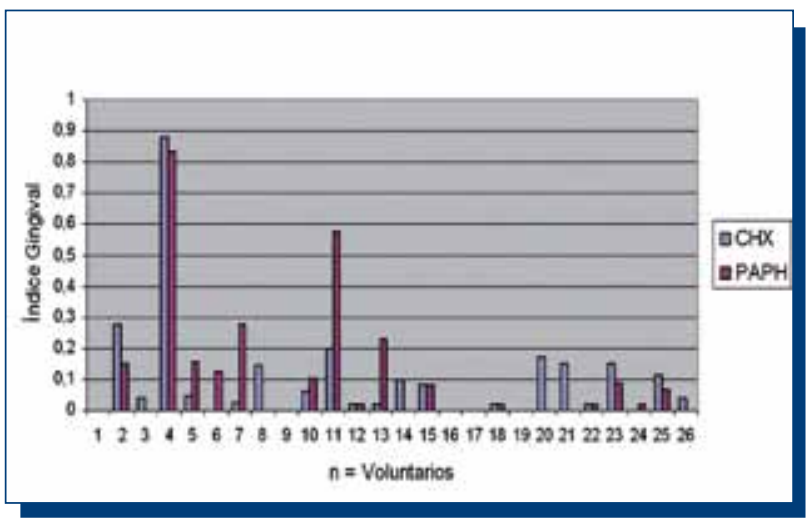

Figura 2. Índice Gingival promedio de cada voluntario después de los cuatro días de tratamiento con cada colutorio. $\mathrm{CHX}$, clorhexidina. PAPH, p-clorofenol alcanforadoperóxido de hidrógeno.

Al realizar el análisis por sexo, arcada, superficie y sector, no se encontraron diferencias entre ambos colutorios, esto esta expresado en la Tabla 2.

Tabla 2. Índice Gingival promedio y t-test para cada subgrupo.

\begin{tabular}{|l|l|c|c|c|c|c|c|c|c|}
\hline \multirow{2}{*}{} & & \multicolumn{2}{|c|}{ Sexo } & \multicolumn{2}{c|}{ Arcada } & \multicolumn{2}{c|}{ Superficie } & \multicolumn{2}{c|}{ Sector } \\
\cline { 2 - 10 } & TOTAL & $\mathbf{M}$ & $\mathbf{H}$ & $\mathbf{S}$ & $\mathbf{I}$ & $\mathbf{V}$ & $\mathbf{P} / \mathbf{L}$ & $\mathbf{A}$ & $\mathbf{P}$ \\
\hline $\mathbf{C H X}$ & 0.099 & 0.063 & 0.118 & 0.084 & 0.112 & 0.111 & 0.087 & 0.144 & 0.045 \\
\hline PAPH & 0.107 & 0.076 & 0.123 & 0.081 & 0.130 & 0.130 & 0.083 & 0.157 & 0.043 \\
\hline $\mathbf{p}$ (t-test) & 0.378 & 0.390 & 0.436 & 0.424 & 0.307 & 0.274 & 0.436 & 0.360 & 0.448 \\
\hline
\end{tabular}

CHX: colutorio de clorhexidina, PAPH: colutorio a base de p-clorofenol alcanforado y peróxido de hidrógeno, M: mujeres, H: hombres, S: arcada superior, I: arcada inferior, V: superficie vestibular, P/L: superficie palatino/lingual, A: sector anterior, P: sector posterior.

El test de Wilcoxon aplicado a los resultados de índice de Placa e índice Gingival después aplicar el tratamiento con ambos colutorios durante cuatro días se puede observar en la Tabla 3.

Tabla 3. Test de Wilcoxon.

\begin{tabular}{|l|c|c|}
\hline & Índice de Placa & Índice Gingival \\
\cline { 2 - 3 } & promedio (DS) & promedio (DS) \\
\hline CHX & $2.200(0.433)$ & $0.099(0.176)$ \\
\hline PAPH & $2.348(0.403)$ & $0.107(0.195)$ \\
\hline
\end{tabular}

Se puede verificar que no existen diferencias estadísticamente significativas en los índices de Placa y Gingival al aplicar el tratamiento con ambos colutorios durante cuatro días.

\section{Acontecimientos Adversos}

Los acontecimientos adversos se presentaron en un total de 35 veces, 27 detectados durante la aplicación de clorhexidina y 7 con p-clorofenol alcanforado-peróxido de hidrógeno, los cuales se detallan en la Tabla 4. Su análisis con el test Chi-cuadrado demostró que no existían diferencias estadísticamente significativas entre los dos colutorios.

Tabla 4. Acontecimientos Adversos (AA) presentados durante la fase de tratamiento con cada colutorio.

\begin{tabular}{|l|c|c|c|c|}
\hline \multirow{2}{*}{ Mal sabor } & \multicolumn{2}{|c|}{ CHX } & \multicolumn{2}{c|}{ PAPH } \\
\cline { 2 - 5 } & $\mathbf{N}^{\circ}$ & $\%$ & $\mathbf{N}^{\circ}$ & $\%$ \\
\hline Ardor & 15 & 57.6 & 2 & 7.7 \\
\hline Náuseas & 5 & 19.2 & 2 & 7.7 \\
\hline Aparición de úlceras & 2 & 7.7 & 1 & 3.8 \\
\hline Parestesia de la lengua & 2 & 7.7 & 0 & 0 \\
\hline Tinciones & 2 & 7.7 & 0 & 0 \\
\hline Boca seca & 0 & 3.8 & 1 & 3.8 \\
\hline TOTAL & $27^{*}$ & 0 & 1 & 3.8 \\
\hline
\end{tabular}

CHX: colutorio de clorhexidina, PAPH: colutorio a base de paramonoclorofenol alcanforado y peróxido de hidrógeno. ${ }^{*} 27$ AA en 17 sujetos $=65.3 \%$ de $n(n=26)$. ${ }^{\star} 7$ AA en 6 sujetos $=23 \%$ de $n(n=26)$.

\section{DISCUSIÓN}

En la literatura no se han descrito estudios acerca de colutorios que contengan p-clorofenol alcanforado y peróxido de hidrógeno, salvo tres investigaciones realizadas en el Departamento de Ciencias Físicas y Químicas de la Facultad de Odontología de la Universidad de Chile, presentados en la IADR-Chile en los años $2006^{(18)}, 2007^{(19)}$ y $2008^{(20)}$. El primero de estos estudios señala que el colutorio disminuye la formación de placa, y al igual que en el presente trabajo, no se hallaron diferencias estadísticamente significativas con la clorhexidina. En aquella oportunidad se evaluó la formación de placa sólo en 24 horas, lo que fue objetado, ya que su estructuración es muy variable dentro de un mismo sujeto en dicho tiempo. Por lo tanto, se decidió utilizar el modelo de formación de placa de novo en cuatro días, descrito en la bibliografía ${ }^{(21,22)}$

El diseño experimental ha sido utilizado por varios autores, entre ellos Sekino ${ }^{(23)}$, Moran ${ }^{(24)}$, Arweiler ${ }^{(25)}$, Herrera ${ }^{(26)}$, Welk ${ }^{(27)}$ y Witt ${ }^{(28)}$. La principal variable medida en estos estudios es el índice de Placa y todos los autores mencionados utilizan el índice de Quigley \& Hein (1962) modificado por Turesky y cols., a excepción de Arweiler ${ }^{(25)}$ que utiliza el índice de Silness \& Löe (1964), por lo tanto, los resultados con respecto al índice de Placa podrían ser comparables. Los valores del índice de Placa alcanzados después del uso de clorhexidina ${ }^{(21,29)}$ y del colutorio se asemejan bastante a los obtenidos por Moran ${ }^{(24)}$ después del uso de cloruro de cetilpiridinio y son mejores que los obtenidos después del uso de cloruro de benzalconio al $0.1 \%$ y $0.05 \%^{(21)}$. Los valores del índice de Placa que obtuvo Moran ${ }^{(24)}$ después del uso de clorhexidina fueron notablemente inferiores, muy distante del valor alcanzado en este estudio. La metodología de Sekino ${ }^{(23,30)}$ difiere de la utilizada en este estudio, ya que los colutorios fueron administrados en los periodos preparatorios y en el periodo de acumulación de placa no había ningún tipo de control de ésta. Shinada y cols., usando un colutorio con polifenoles, encuentra una reducción del índice de Placa un poco mayor, pero sólo en tres días de tratamiento(31).

En este estudio la clorhexidina fue significativamente más eficaz en la inhibición del crecimiento de placa en la arcada superior en las superficies vestibulares y en el sector posterior, por otra parte el colutorio en evaluación fue significativamente más eficaz en la inhibición del crecimiento de placa en las superficies linguales/palatinas. Sin embargo, aún no tenemos explicación para este hecho. También cabe mencionar que de todos los estudios citados anteriormente en ninguno se analiza el índice de Placa por arcada, superficie y sector.

Otras investigaciones analizan la efectividad antiplaca y antigingivitis de colutorios que contienen triclosán y timol ${ }^{(30)}$, pero no se describen enjuagatorios que contengan p-clorofenol. En el estudio realizado el 2008 se analizó la eficacia antimicrobiana in vivo e in vitro, específicamente sobre cepas de Porphyromonas gingivalis y Streptococcus mutans y se obtuvo inhibición del crecimiento de ambas cepas $^{(20)}$. En Agar Columbia se observó inhibición total del crecimiento bacteriano de Porphyromonas gingivalis posterior al uso del colutorio e inhibición dependiente de la concentración cuando fueron incubadas en diluciones al 50\%, 90\%, 95\% y 100\%. En Agar TYCSB se observó inhibición parcial del crecimiento bacteriano de Streptococcus mutans. Esto es una clara evidencia de que el colutorio posee actividad antibacteriana $^{(20)}$.

Las referencias bibliográficas del uso de la clorhexidina en sus diferentes presentaciones ha sido ampliamente documentada, quedando claramente establecido que la clorhexidina al $0.12 \%$ es el "gold standard" en cuanto a colutorios antiplaca y antigingivitis, es por ello que fue utilizada como control positivo(1). Se decidió no incluir un grupo control negativo, ya que se conocía que la formulación en base a p-clorofenol alcanforado y peróxido de hidrógeno efectivamente posee actividad antiplaca ${ }^{(18)}$. De todos los trabajos de formación de placa en 4 días mencionados anteriormente, sólo el de Herrera( ${ }^{(26)}$ incluye también la medición del índice Gingival (Löe-Silness 1963), sin embargo, los voluntarios de dicho estudio partieron con cierto nivel basal de gingivitis, por lo tanto los datos no son comparables a los de este estudio. Otros evalúan la eficacia antigingivitis de los colutorios en periodos de 2 semanas $^{(30,32)}$ y prácticamente la totalidad de los estudios que analizan algún mecanismo contra la gingivitis tienen una duración mínima de la fase experimental de 3 semanas, la Asociación Dental Americana (ADA) recomienda trabajos clínicos de 6 meses de duración para el establecimiento de la eficacia a largo plazo de los colutorios ${ }^{(22)}$. Por lo tanto, se considera en este estudio el índice de Placa como la variable de mayor importancia, no hallándose diferencias estadísticamente significativas en la eficacia antiplaca entre 
la clorhexidina $0.12 \%$ y el colutorio a base de p-clorofenol alcanforado y peróxido de hidrógeno. Dada la sustantividad que posee la clorhexidina, puede ser administrada cada 12 horas y mantener su capacidad para retardar o prevenir la formación de placa ${ }^{(23)}$. La posología depende del tiempo que los compuestos se mantengan activos en boca, por lo tanto, es imprescindible conocer la vida media del colutorio, para ajustar la dosis, de esta manera tal vez se podrían obtener mejores resultados.

Un aspecto que podría causar controversia es la citotoxicidad que pudieran presentar el p-clorofenol alcanforado al $0.03 \%$ y peróxido de hidrógeno al $0.04 \%$. En el caso del derivado fenólico la concentración utilizada está diluída aproximadamente 1.000 veces con respecto a la cantidad que tiene este elemento en otras presentaciones donde se presenta al $33 \%$. El otro principio activo está diluído aproximadamente 75 y 875 veces con respecto a lo utilizado en irrigaciones $(3 \%)$ y en agentes blanqueadores $\left(\mathrm{H}_{2} \mathrm{O}_{2} \text { al } 35 \%\right)^{(7)}$.

En este estudio sólo se han utilizado pacientes sanos y no se han presentado acontecimientos adversos relevantes, es más, se han presentado en una menor cantidad en comparación con los que ha presentado la clorhexidina ${ }^{(32)}$.

\section{CONCLUSIONES}

El desarrollo de este colutorio apunta a tener un coadyuvante de muy bajo costo para el control de la placa microbiana y de la inflamación gingival, si bien los autores reconocen la necesidad de realizar más investigación, consideran que tiene las características para ser una alternativa en esas situaciones.

\section{REFERENCIAS BIBLIOGRÁFICAS}

1. Jones CG. Chlorhexidine: is it still the gold standard? Periodontol 2000,1997; 15:55-62.

2. Harrison JW, Madonia JV. The toxicity of parachlorophenol. Oral Surg Oral Med Oral Pathol, 1971; 1: 90-99.

3. Llamas R, Segura J. In vitro effect of parachlorophenol and camphorated parachlorophenol on macrophages. J Endod,1997;23:728-730.

4. Photios JM. The effect of metacresylacetate and camphorated chlorophenol on the dental pulp. Oral Surg Oral Med Oral Pathol,1968; 26(6):848-855.

5. Harrison JW, Bellizzi R, Osetek EM. The clinical toxicity of endodontic medicaments. J Endod,1979 Feb;5(2):42-47.

6. Thomas EL, Milligan TW, Joyner RE, Jefferson MM. Antibacterial activity of hydrogen peroxide and the lactoperoxidase-hydrogen peroxide-thiocyanate system against oral streptococci. Infect Immun, 1994 Feb; 62(2):529-535.

7. Agencia para Sustancias Tóxicas y el Registro de Enfermedades (ATSDR). Gestión de incidentes de materiales peligrosos. Volumen III - Gestión de las directrices médicas de exposiciones químicas aguda: peróxido de hidrógeno. Atlanta, GA: EE.UU. Departamento de Salud y Servicios Humanos, Servicio de Salud Pública, 2002

8. Marshall MV. Hydrogen peroxide: a review of its use in dentistry. $J$ Periodontol,1995;66(9):786-796.

9. Murray P, Baron E, Pfaller M, Tenover F, Yolken R. Manual of clinical microbiology. $6^{\text {th }}$ edition. ASM Press, 1995.

10. Yévenes I, Jaña D, Torres CG, Oyarzún A, Rivera S, Galván T. Efecto de colutorio fenolalcanforado sobre células de la mucosa oral. Libro de resúmenes XXII Reunión Anual IADR Chile,2009:41.

11. Reis SRA, Espírito Santo AR, Andrade MGS, Sadigursky M. Cytologic alterations in the oral mucosa after chronic exposure to ethanol. Braz Oral Res,2006;20(2):97-102.

12. Harrison JW, Madonia JV. The toxicity of parachlorophenol. Oral Surg Oral Med Oral Pathol,1971;32(1):90-99.

13. Soekanto A, Kasugai S, Mataki S, Ohya K, Ogura H. Toxicity of camphorated phenol and camphorated parachlorophenol in dental pulp cell culture. J Endod, June 1996;22(6):284-286.

14. Davoren M, Fogarty AM. In vitro cytotoxicity assessment of the biocidal agents sodium o-phenylphenol, sodium o-benzyl-p-chlorophenol, and sodium p-tertiary amylphenol using established fish cell lines. Toxicol In Vitro,2006 Oct;20(7):1190-1201.

15. Mahony C, Felter SP, Mc Millan DA. An exposure-based risk assessment approach to confirm the safety of hydrogen peroxide for use in home tooth bleaching. Regul Toxicol Pharmacol,2006 Mar;44(2):75-82.

16. Loe H, Silness J. Periodontal disease in pregnancy. Acta Odontol Scand, 1963:21:533-538

17. Turesky S, Gilmore ND, Glickman I. Reduced plaque formation by the chlorometyl analogue of vitamine C. J Periodontol,1970;41:41-43.

18. Yévenes I, Rivera S, Neira M, Jaña D y cols. Efecto comparativo antiplaca de colutorio a base de paraclorofenol, alcanfor y peróxido de hidrógeno con colutorio de clorhexidina al $0.12 \%$. Libro de resúmenes XIX Reunión Anual IADR Chile,2006;72:29.
19. Yévenes I, Palma P, Jaña D, Espinoza Py cols. Efecto químico microbiológico en saliva por uso de colutorio antiséptico. Libro de resúmenes $X X$ Reunión Anual IADR Chile,2007;148:28.

20. Yévenes I, Palma P, Gajardo M, Neira M, Galván T. Actividad antimicrobiana in vivo e in vitro de un colutorio a base de paraclorofenol, alcanfor y peróxido de hidrógeno. XXI Reunión Anual IADR Chile,2008.

21. Barnett $M$. The rol of therapeutic antimicrobial mouthrinses in clinical practice: control of supragingival plaque and gingivitis. J Am Dent Assoc, 2003;134:699-704.

22. Moran J, Addy M, Newcombe R. A4-day plaque regrowth study comparing an essential oil mouthrinse with a triclosan mouthrinse. J Clin Periodontol, 1997:24:636-639.

23. Sekino S, Ramberg P, Uzel NG, Socransky S. The effect of clorhexidine regimen on de novo plaque formation. J Clin Periodontol,2004;31:609-614.

24. Moran J, Addy M, Jackson R, Newcombe RG. Comparative effects of quaternary ammonium mouthrinses on 4-day plaque regrowth. $J$ Clin Periodontol,2000;27:37-40.

25. Arweiler NB, Boehnke N, Sculean A, Hellwig E, Auschill TM. Differences in efficacy of two commercial $0.2 \%$ chlorhexidine mouthrinse solutions: a 4-day plaque re-growth study. J Clin Periodontol,2006; 33:334-339.

26. Herrera D, Santos S, Ferrús J, Barbieri G, Trombelli L, Sanz M. Efficacy of a $0.15 \%$ benzydamine hydrochloride and $0.05 \%$ cetylpyridinium chloride mouth rinse on 4-day de novo plaque formation. $J$ Clin Periodontol,2005 Jun;32(6):595-603.

27. Welk A, Splieth $\mathrm{CH}$, Schmidt-Martens $\mathrm{G}$ y cols. The effect of a polyhexamethylene biguanide mouthrinse compared with a triclosan rinse and a chlorhexidine rinse on bacterial counts and 4-day plaque regrowth. J Clin Periodontol,2005 May;32(5):499-505.

28. Witt J, Ramji N, Gibb R, Dunavent J, Flood J, Barnes J. Antibacterial and antiplaque effects of a novel, alcohol-free oral rinse with cetylpyridinium chloride. J Contemp Dent Pract,2005;6:1-9.

29. Hasson H, Ismail A, Neiva G. Home-based chemically-induced whitening of teeth in adults. Cochrane Database of Systematic Reviews,2006, Issue 4. 30. Sekino S, Ramberg P. The effect of a mouthrinse containing phenolic compounds on plaque formation and developing gingivitis. J Clin Periodontol,2005;32(10):1083-1088.

31. Shinada K, Tagashira M, Watanabe H, Sopapornamorn P, Kanayama A, Kanda T, Ikeda M, Kawaguchi Y. Hop bract polyphenols reduced three-day dental plaque regrowth. J Dent Res,2007;86(9):848-851.

32. Grundmann LJ, Timmerman MF, Ljzerman Y, van der Weijden GA. Stain, plaque and gingivitis reduction by combining chlorhexidine and peroxyborate. J Clin Periodontol,2000;27:9-15. 